\section{Estado nutricional de crianças indígenas Guarani nos estados do Rio de Janeiro e São Paulo, Brasil}

\author{
Nutritional status of Guarani indigenous children in \\ the States of Rio de Janeiro and São Paulo, Brazil \\ Estado nutricional de niños indígenas Guaraní en
los estados de Río de Janeiro y São Paulo, Brasil
}

\begin{abstract}
This article reports the results of a nutritional survey among Guarani indigenous children $<5$ years of age in the States of Rio de Janeiro and São Paulo, Brazil. Prevalence rates for malnutrition according to various anthropometric indices were $50.4 \%$ (low stature-for-age), $7.9 \%$ (low weight-for-age), and $0.8 \%$ (low weight-for-stature). Prevalence of stunting in Guarani children was 96\% higher than for indigenous children in Brazil as a whole (25.7\%) and 7.2 times that in children from the general population (7\%). Prevalence of anemia was 65.2\%, 3.1 times higher than in non-indigenous children (20.9\%). The study highlights the high prevalence of chronic undernutrition and anemia in Guarani children and calls attention to serious inequalities in health and nutrition that affect indigenous children in Brazil.
\end{abstract}

Indigenous Population; Child Health; Nutrition Surveys; Nutritional Status
Carla Tatiana Garcia Barreto 1 Andrey Moreira Cardoso 1 Carlos E. A. Coimbra Jr. ${ }^{1}$

\section{Resumo}

O presente estudo reporta os resultados de inquérito nutricional de crianças indígenas Guara$n i<5$ anos de idade nos estados do Rio de Janeiro e São Paulo, Brasil. As prevalências de desnutrição, segundo vários índices antropométricos foram de: 50,4\% (baixa estatura para idade), 7,9\% (baixo peso para idade) e 0,8\% (baixo peso para estatura). O déficit de estatura verificado na criança Guarani foi $96 \%$ maior do que nas crianças indígenas no Brasil (25,7\%) e 7,2 vezes do que o registrado nas crianças da população geral (7\%). A prevalência de anemia foi de 65,2\%, 3,1 vezes a prevalência correspondente nas crianças não indígenas (20,9\%). Este estudo destaca elevadas prevalências de desnutrição crônica e anemia na criança Guarani, e põe em evidência as graves desigualdades em saúde e nutrição que afetam a criança indígena no país.

População Indígena; Saúde da Criança; Inquéritos Nutricionais; Estado Nutricional 


\section{Introdução}

O estado nutricional de crianças é determinado por fatores biológicos e socioeconômicos, sendo a desnutrição infantil considerada um indicador de desigualdade social 1. Recentemente, o I Inquérito Nacional de Saúde e Nutrição dos Povos Indígenas no Brasil 2,3,4 reafirmou em escala nacional a importância da desnutrição no perfil de saúde da criança indígena no país, o que já vinha sendo sinalizado por diversos levantamentos que enfocaram etnias específicas 5,6,7. Tanto no Inquérito Nacional quanto nos estudos de comunidade são observadas elevadas prevalências de desnutrição crônica e anemia, comumente superando as magnitudes descritas para a população infantil brasileira geral.

Em relação ao povo indígena Guarani que vive no Brasil, estudos recentes têm evidenciado precárias condições de saúde. São elevadas as taxas de hospitalização e óbito de crianças por doenças respiratórias agudas (DRA) 8,9,10, assim como as prevalências de parasitoses intestinais e poliparasitismo ${ }^{11}$. No Rio de Janeiro, foi reportado um aglomerado de casos de hanseníase, que incluiu caso multibacilar e em jovem $<15$ anos 12 . O perfil de saúde Guarani delineado pelos trabalhos existentes sugere fortemente que a população vive em condições de precariedade e tem limitações no acesso e na qualidade da atenção à saúde.

Tendo em vista a sociodiversidade dos povos indígenas no país e a disponibilidade, pela primeira vez, de dados sobre o estado nutricional indígena em âmbito nacional e regional, o objetivo deste artigo é descrever e comparar os resultados de um inquérito nutricional de crianças Guarani $<5$ anos de idade, residentes em aldeias nos estados do Rio de Janeiro e São Paulo, conduzido por meio de metodologia padronizada, aos resultados do Inquérito Nacional ${ }^{3}$.

\section{População e métodos}

Foi realizado um inquérito nutricional e de anemia em crianças indígenas Guarani < 5 anos, residentes em 5 aldeias no Estado do Rio de Janeiro (Sapukai, Parati-Mirim, Araponga, Sítio Rio Pequeno e Mamanguá) e 1 aldeia no litoral norte do Estado de São Paulo (Boa Vista).

Essas comunidades Guarani vivem em aldeias litorâneas próximas a cidades e mantêm contato intenso com a população do entorno 13 . Em geral, consomem alimentos industrializados, oriundos de compras. Há também os recursos provenientes de partilha entre famílias, doações e, raramente, caça ou coleta 14 .
O levantamento dos dados ocorreu de novembro de 2008 a março de 2009. O censo das aldeias foi obtido no Sistema de Informação da Atenção à Saúde Indígena (SIASI) local, e atualizado por consulta ao cadastro de famílias dos agentes indígenas de saúde e aos espelhos dos cartões vacinais em posse das Equipes Multidisciplinares de Saúde Indígena.

Os pesquisadores foram treinados e padronizados em antropometria em oficina, tendo sido utilizados instrumentos e técnicas recomendados pela Organização Mundial da Saúde (OMS) 15 .

Foram calculados os índices antropométricos estatura para idade (E/I), peso para idade $(\mathrm{P} / \mathrm{I})$ e peso para estatura $(\mathrm{P} / \mathrm{E})$, baseando-se na população referência da OMS 15. Considerou-se desnutrição o escore $\mathrm{z}<-2$ desvios-padrão (DP) para um dos três índices antropométricos, assim como risco nutricional o escore $\mathrm{z} \geq-2 \mathrm{DP} \mathrm{e}<-1 \mathrm{DP}$. $\mathrm{O}$ sobrepeso foi definido pelo escore $\mathrm{z} \geq+2 \mathrm{DP}$ para o índice $\mathrm{P} / \mathrm{E}$.

A hemoglobina $(\mathrm{Hb})$ foi dosada em sangue da extremidade digital em crianças de 6 a 59 meses, com hemoglobinômetro portátil HemoCue (Ångelholm, Suécia), modelo Hb201+. Considerouse anemia $\mathrm{Hb}<11 \mathrm{~g} / \mathrm{dL}$ e anemia moderada $\mathrm{Hb}$ entre 7 e 9,9g/dL 16 . As análises foram conduzidas no programa Stata, versão 9.0 (Stata Corp., College Station, Estados Unidos).

A pesquisa foi aprovada pelo Comitê de Ética em Pesquisa/Ensp/Fiocruz (protocolo no 130/05), pela Comissão Nacional de Ética em Pesquisa (protocolo no 154/2006), pela Fundação Nacional do Índio (no 23/CGEP/07), pelas lideranças indígenas e pelo responsável por cada criança.

\section{Resultados}

A população alvo totalizava 143 crianças, mas 15 (10,5\%) não foram avaliadas (13 por ausência e 2 por recusa). A antropometria foi realizada em 128 $(89,5 \%)$ crianças e a dosagem de hemoglobina em 115 (89,8\%).

As prevalências de desnutrição pelos índices E/I, P/I e P/E foram, respectivamente, 50,4\% (M: 52,4\%; F: 48,4\%), 7,9\% (M: 10,9\%; F: 4,8\%) e 0,8\% (M: 0,0\%; F: 1,6\%). A prevalência de sobrepeso foi de 4,0\% (M: 7,8\%; F: 0,0\%). As prevalências de distúrbios nutricionais segundo faixas etárias são apresentadas na Tabela 1.

A prevalência de anemia foi de $65,2 \%$, e $38.3 \%$ apresentaram anemia moderada. As prevalências anemia segundo sexo e faixas etárias são apresentadas na Tabela 2 . 


\section{Tabela 1}

Prevalências globais e por faixa etária de desnutrição, risco nutricional e sobrepeso segundo os índices estatura/idade (E/I), peso para idade (P/I) e peso para estatura (P/E).

\begin{tabular}{|c|c|c|c|c|c|c|c|c|c|}
\hline \multirow[t]{2}{*}{ Faixa etária (meses) } & \multicolumn{5}{|c|}{ Guarani (2008/2009) } & \multicolumn{2}{|c|}{ Inquérito indígena $(2008 / 2009) 2$} & \multicolumn{2}{|c|}{ PNDS 200617} \\
\hline & $\mathrm{n}$ & Déficit & Risco & Adequado & Excesso & Déficit & Excesso & Déficit & Excesso \\
\hline \multicolumn{10}{|c|}{ Prevalência escore z E/I } \\
\hline $0-11$ & 20 & 45,0 & 25,0 & 30,0 & - & - & - & 4,8 & - \\
\hline $0-5$ & 11 & 45,5 & 18,2 & 36,4 & - & 9,2 & - & - & - \\
\hline $6-11$ & 9 & 44,4 & 33,3 & 22,2 & - & 14,9 & - & - & - \\
\hline $12-23$ & 25 & 60,0 & 20,0 & 20,0 & - & 31,5 & - & 12,3 & - \\
\hline $24-35$ & 26 & 53,8 & 42,3 & 3,8 & - & 32,7 & - & 7,1 & - \\
\hline $36-47$ & 27 & 55,6 & 37,0 & 7,4 & - & 28,3 & - & 6,3 & - \\
\hline $48-59$ & 27 & 37,0 & 55,6 & 7,4 & - & 25,2 & - & 4,7 & - \\
\hline Total & 125 & 50,4 & 36,8 & 12,8 & - & 25,7 & - & 7,0 & - \\
\hline \multicolumn{10}{|l|}{ Prevalência escore z P/l } \\
\hline $0-11$ & 20 & 10,0 & 20,0 & 70,0 & - & - & - & 2,9 & - \\
\hline $0-5$ & 11 & 9,1 & 18,2 & 72,7 & - & 4,0 & - & - & - \\
\hline 6-11 & 9 & 11,1 & 22,2 & 66,7 & - & 7,3 & - & - & - \\
\hline $12-23$ & 25 & 16,0 & 48,0 & 36,0 & - & 7,5 & - & 2,2 & - \\
\hline $24-35$ & 27 & 7,4 & 33,3 & 59,3 & - & 6,1 & - & 1,2 & - \\
\hline $36-47$ & 28 & 3,6 & 28,6 & 67,9 & - & 5,1 & - & 1,5 & - \\
\hline $48-59$ & 27 & 3,7 & 7,4 & 88,9 & - & 5,1 & - & 1,4 & - \\
\hline Total & 127 & 7,9 & 27,6 & 64,6 & - & 5,9 & - & 1,9 & - \\
\hline \multicolumn{10}{|c|}{ Prevalência escore z P/E } \\
\hline $0-11$ & 20 & 5,0 & - & 85,0 & 10,0 & - & - & 2,9 & 6,9 \\
\hline $0-5$ & 11 & 9,1 & - & 72,7 & 18,2 & 2,7 & - & - & - \\
\hline $6-11$ & 9 & - & - & 100,0 & - & 2,6 & - & - & - \\
\hline $12-23$ & 25 & - & 20,0 & 80,0 & - & 2,0 & - & 2,5 & 6,1 \\
\hline $24-35$ & 26 & - & - & 100,0 & - & 0,4 & - & 0,1 & 7,1 \\
\hline $36-47$ & 27 & - & - & 96,3 & 3,7 & 0,7 & - & 0,7 & 9,7 \\
\hline $48-59$ & 27 & - & - & 92,6 & 7,4 & 0,6 & - & 0,9 & 6,4 \\
\hline Total & 126 & 0,8 & 4,0 & 91,3 & 4,0 & 1,3 & - & 1,4 & 7,3 \\
\hline
\end{tabular}

PNDS: Pesquisa Nacional de Demografia e Saúde da Criança e da Mulher.

Tabela 2

Prevalência de anemia global, por sexo e faixa etária, em crianças indígenas Guarani.

\begin{tabular}{lcccccc}
\hline Faixa etária (meses) & \multicolumn{2}{c}{ Masculino } & \multicolumn{2}{c}{ Feminino } & \multicolumn{2}{c}{ Global } \\
& $\mathbf{n}$ & $\%$ & $\mathbf{n}$ & $\%$ & $\mathbf{n}$ & $\%$ \\
\hline $6-11$ & 4 & 100,0 & 5 & 80,0 & 9 & 88,9 \\
$12-23$ & 9 & 66,7 & 15 & 80,0 & 24 & 75,0 \\
$24-35$ & 12 & 83,3 & 15 & 53,3 & 27 & 66,7 \\
$36-47$ & 16 & 62,5 & 11 & 36,4 & 27 & 51,9 \\
$48-59$ & 19 & 68,4 & 8 & 37,5 & 27 & 59,3 \\
Total & 60 & 71,7 & 54 & 57,4 & 115 & 65,2 \\
\hline
\end{tabular}




\section{Discussão}

As aldeias investigadas estão localizadas em estados do Sudeste, cujos Índices de Desenvolvimento Humano estão entre os maiores do país. Apesar disso, os resultados deste estudo evidenciam expressivas disparidades nos indicadores nutricionais e de anemia entre os indígenas e os não indígenas.

$O$ déficit de estatura Guarani atinge mais da metade das crianças investigadas, sendo 7,2 e 9,0 vezes as prevalências correspondentes em crianças não indígenas no Brasil (7\%) e no Sudeste $(5,6 \%)$, respectivamente 17 . O déficit estatural Guarani supera até mesmo o observado nas crianças não indígenas do Norte $(14,7 \%)$, que registra a maior prevalência de desnutrição por este indicador no país 17.

A prevalência de déficit de P/I superou em mais de 4 vezes a prevalência nas crianças não indígenas no Brasil (1,9\%), sendo 5,6 vezes a prevalência no Sudeste. Por outro lado, a prevalência de baixo P/E Guarani (0,8\%) foi menor do que a observada nas crianças brasileiras não indígenas $(1,4 \%)$, assim como o excesso de peso (Guarani: 4\%; não indígena: 7,3\%) 17 .

A prevalência de anemia Guarani $(65,2 \%)$ é considerada grave, superando 3,1 vezes a prevalência em crianças não indígenas no Brasil (20,9\%) e em 6,3 a da Região Norte, que registra a maior prevalência nacional 17 .

As iniquidades no perfil nutricional Guarani permanecem, mesmo quando são comparadas ao contexto indígena no Brasil 3,4. O déficit de estatura supera em 2 vezes a prevalência média correspondente nos indígenas no país $(25,7 \%)$, sendo de 1,2 (Norte: 40,8\%) a 3,6 (Nordeste: $13,9 \%$ ) vezes as prevalências indígenas nas diferentes regiões 3 . O déficit de P/I Guarani só foi superado pelo observado nos indígenas do Norte $(11,4 \%)$, tendo sido 2 vezes a prevalência verificada nos indígenas do Sul/Sudeste, ao passo que a prevalência de baixo P/E Guarani foi inferior às prevalências médias nos indígenas de todas as regiões.

A prevalência de anemia Guarani também supera os valores médios para o conjunto das crianças indígenas no Brasil $(51,2 \%)$ e para as residentes no Sul/Sudeste (48\%), sendo próxima à reportada para as crianças indígenas do Norte $(66,4 \%) 4$

A desnutrição tem sido associada ao risco de morte por algumas doenças infecciosas, sendo as principais delas as DRA e as diarreias 18. Esses agravos estão configurados como as principais causas de hospitalização e óbito de crianças Guarani $<5$ anos no Sul e Sudeste do Brasil 8,9,10, sugerindo que esta população encontra-se em um círculo infecção-desnutrição.

As prevalências de desnutrição e anemia verificadas neste estudo são expressivas em comparação à situação indígena e não indígena no país. Os resultados reforçam a necessidade de investimentos na atenção materno-infantil entre os Guarani. Medidas de saneamento básico e estratégias de segurança alimentar e subsistência devem ser implementadas, com ampla discussão com as comunidades.

\section{Resumen}

Este estudio presenta los resultados de una encuesta nutricional realizada entre niños indígenas Guara$n i ́<5$ años de edad en los estados de Río de Janeiro y São Paulo, Brasil. La frecuencia de desnutrición, según varios índices antropométricos fue: $50,4 \%$ (baja tallapara-edad), 7,9\% (bajo peso-para-edad) y 0,8\% (bajo peso-para-talla). El retraso del crecimiento verificado en niños Guaraní fue un 96\% mayor que lo que se observa entre los niños indígenas en Brasil (25,7\%) y 7,2 veces a la de niños en la población general (7\%). La pre- valencia de anemia fue de un 65,2\%; 3,1 veces la prevalencia correspondiente en niños no indígenas (20,9\%). Este estudio destaca la alta prevalencia de desnutrición crónica y anemia en los niños Guaraní y pone en evidencia las graves desigualdades en salud y nutrición que afectan a los niños indígenas en el país.

Población Indígena; Salud del Niño; Encuestas Nutricionales; Estado Nutricional 


\section{Colaboradores}

C. T. G. Barreto e A. M. Cardoso participaram na concepção do projeto, coleta e interpretação dos dados, redação do artigo e aprovação final da versão a ser publicada. C. E. A. Coimbra Jr. participou na concepção do projeto, interpretação dos dados, redação do artigo e aprovação final da versão a ser publicada.

\section{Agradecimentos}

Agradecemos o apoio financeiro do CNPq, DECIT/ MS e FAPERJ (Editais MCT-CNPq/MS-SCTIE-DECIT no 26/2006 - processo 409677/2006-0; MCT/CNPq 02/2006 Universal - processo 479224/2006-4; FAPERJE-26, processo N0170.504/2007), às Equipes Multidisciplinares de Saúde Indígena dos Polos Base de Angra dos Reis e Litoral Norte de São Paulo, e aos indígenas Guarani, que apoiaram o trabalho como possibilidade de contribuição para a saúde de suas crianças.

\section{Referências}

1. Monteiro CA, Benicio MHD'A, Iunes RF, Gouveia NC, Cardoso MAA. Evolução da desnutrição infantil. In: Monteiro CA, organizador. Velhos e novos males da saúde no Brasil. 2a Ed. São Paulo: Editora Hucitec; 2000. p. 93-114.

2. Coimbra Jr. CEA, Santos RV, Welch JR, Cardoso AM, Souza MC, Garnelo L, et al. The First National Survey of Indigenous People's Health and Nutrition in Brazil: rationale, methodology, and overview of results. BMC Public Health 2013; 13:52.

3. Horta BL, Santos RV, Welch JR, Cardoso AM, Santos JV, Assis AMO, et al. Nutritional status of indigenous children: findings from the First National Survey of Indigenous Peoples Health and Nutrition in Brazil. Int J Equity Health 2013; 12:23.

4. Leite MS, Cardoso AM, Coimbra Jr. CEA, Welch JR, Gugelmin AS, Lira PCI, et al. Prevalence of anemia and associated factors among indigenous children in Brazil: results from the First National Survey of Indigenous People's Health and Nutrition. Nutr J 2013; 12:69.

5. Kühl AM, Corso ACT, Leite MS, Bastos JL. Perfil nutricional e fatores associados à ocorrência de desnutrição entre crianças indígenas Kaingáng da Terra Indígena de Mangueirinha, Paraná, Brasil. Cad Saúde Pública 2009; 25:409-20.
6. Menegolla IA, Drachler ML, Rodrigues IH, Schwingel LR, Scapinello E, Pedroso MB, et al. Estado nutricional e fatores associados à estatura de crianças da Terra Indígena Guarita, Sul do Brasil. Cad Saúde Pública 2006; 22:395-406.

7. Ferreira AA, Welch JR, Santos RV, Gugelmin AS, Coimbra Jr. CEA. Nutritional status and growth of indigenous Xavante children, Central Brazil. Nutr J 2012; 11:3.

8. Cardoso AM, Coimbra Jr. CEA, Tavares FG. Morbidade hospitalar indígena Guarani no Sul e Sudeste do Brasil. Rev Bras Epidemiol 2010; 13:21-34.

9. Cardoso AM, Coimbra Jr. CEA, Barreto CTG, Werneck GL, Santos RV. Mortality among Indians in Southeastern and Southern Brazil. Cad Saúde Pública 2011; 27 Suppl 2:S222-36.

10. Cardoso AM, Coimbra Jr. CEA, Werneck GL. Risk factors for hospital admission due to acute lower respiratory tract infection in Guarani indigenous children in southern Brazil: a population-based case-control study. Trop Med Int Health 2013; 18:596-607. 
11. Brandelli CLC, De Carli GA, Macedo AJ, Tasca T. Intestinal parasitism and socio-environmental factors among Mbyá-Guarani Indians, Porto Alegre, Rio Grande do Sul, Brazil. Rev Inst Med Trop São Paulo 2012; 54:119-22.

12. Nery JAC, Pimentel MIF, Lyra MR, Sohsten BLV, Marinho DP, Périssé ARS. Detection of clusters of leprosy cases among Guarani Indians in the southern region of the state of Rio de Janeiro, Brazil. Rev Soc Brasil Med Trop 2012; 45:538-9.

13. Ladeira MI. O caminhar sob a luz: território Mbya à beira do oceano. São Paulo: Editora UNESP; 2007.

14. Pissolato E. A duração da pessoa: mobilidade, parentesco e xamanismo Mbyá (Guarani). São Paulo: Editora UNESP; 2007.

15. World Health Organization. Physical status: the use and interpretation of anthropometry. Geneva: World Health Organization; 1995.
16. World Health Organization. Iron deficiency anaemia assessment, prevention, and control: a guide for programme managers. Geneva: World Health Organization; 2001

17. Ministério da Saúde. Pesquisa Nacional de Demografia e Saúde da Criança e da Mulher-PNDS 2006: dimensões do processo reprodutivo e da saúde da criança. Brasília: Ministério da Saúde; 2009.

18. Rice AL, Sacco L, Hyder A, Black RE. Malnutrition as an underlying cause of childhood deaths associated with infectious diseases in developing countries. Bull World Health Organ 2000; 78:1207-21.

Recebido em 19/Jun/2013

Versão final reapresentada em 27/Nov/2013

Aprovado em 12/Dez/2013 